\title{
Classical dynamics and localization of resonances in the high energy region of the hydrogen atom in crossed fields
}

\author{
Frank Schweiner, Jörg Main, Holger Cartarius, and Günter Wunner \\ Institut für Theoretische Physik 1, Universität Stuttgart, 70550 Stuttgart, Germany
}

(Dated: December 11, 2014)

\begin{abstract}
When superimposing the potentials of external fields on the Coulomb potential of the hydrogen atom a saddle point appears, which is called the Stark saddle point. For energies slightly above the saddle point energy one can find classical orbits, which are located in the vicinity of this point. We follow those so-called quasi-Penning orbits to high energies and field strengths observing structural changes and uncovering their bifurcation behavior. By plotting the stability behavior of those orbits against energy and field strength the appearance of a stability apex is reported. A cusp bifurcation, located in the vicinity of the apex, will be investigated in detail. In this cusp bifurcation another orbit of similar shape is found, which becomes completely stable in the observed region of positive energy, i.e., in a region of parameter space, where the Kepler-like orbits located around the nucleus are already unstable. By quantum-mechanically exact calculations we prove the existence of signatures in quantum spectra belonging to those orbits. Husimi distributions are used to compare quantum-Poincaré sections with the extension of the classical torus structure around the orbits. Since periodic orbit theory predicts that each classical periodic orbit contributes an oscillating term to photoabsorption spectra, we finally give an estimation for future experiments, which could verify the existence of the stable orbits.
\end{abstract}

PACS numbers: 32.80.-t, 32.60.+i, 05.45.-a

\section{INTRODUCTION}

The hydrogen atom in crossed electric and magnetic fields is a simple example of a non-integrable physical system, which has up to now been investigated for almost one hundred years, theoretically [1/5] as well as experimentally [3]. Furthermore it has been used for studies on phenomena like Ericson fluctuations [8, 15] or Arnol'd diffusion [6]. Even quantum dots [16] and excitons [17] in condensed matter physics can be explored using the findings attained from the hydrogen atom in crossed fields.

One of the major purposes in recent decades was to uncover how chaos, which can be observed in a classical treatment [18, 19, shows itself in quantum spectra, since the Schrödinger equation is, due to its linearity, not capable of producing chaotic behavior [5, 20, 26. Nevertheless new phenomena occur in crossed fields: the so called quasi-Penning resonances [27. From a classical point of view those resonances describe a movement of the electron which is localized around a saddle point in the potential, the Stark saddle point. The possible appearance of wave functions localized far away from the nucleus led to a series of further investigations [27 [32, since they can play an important role in the ionization process. Transition state theory predicts classical orbits localized around the Stark saddle point and states that all ionizing orbits have to pass the vicinity of this point 33 36. Even though Clark et al. 27] already proved their existence in 1985, it was not until 2009 that first signatures of those orbits were found in calculated quantum spectra [37. The classical stability behavior of the quasi-Penning orbits at high energies and field strengths has first been investigated by Flöthmann in 1994 [38], who uncovered a stability apex in parameter space, if the stability change of the orbits is illustrated as a function of both parameters.

It is the purpose of this paper to perform more precise calculations on the stability of those orbits and to uncover the bifurcation behavior as well as the processes taking place around the stability apex. We report the existence of a cusp bifurcation, which appears in the vicinity of the stability apex and involves another orbit of similar shape. This orbit, becoming completely stable in a specific area of parameter space, is the starting point for semiclassical and quantum mechanical calculations. We will demonstrate that signatures of those orbits at high energies and field strengths can be found in accurately calculated quantum spectra.

The paper is organized as follows. In Sec. II the system is introduced, and a scaling of parameters as well as a regularization of coordinates are carried out. A comparison between exceptional points and a classical cusp bifurcation is drawn. The classical stability of the quasi-Penning resonances and their bifurcation behavior at high energies and field strengths are presented in Sec. [III. An introduction in the semiclassical and exact quantum mechanical calculations performed is given in Sec. IV] before according results are discussed in Sec. V. In Sec. VI a short summary is given and conclusions are drawn. 


\section{CLASSICAL CALCULATIONS}

\section{A. Hamiltonian, monodromy matrix and regularized coordinates}

The Hamiltonian of a hydrogen atom in a constant electric field $\boldsymbol{F}=F \hat{e}_{x}$ and a constant magnetic field $\boldsymbol{B}=$ $B \hat{e}_{z}$ reads in atomic units (Hartree units with lengths, energies, electric and magnetic field strengths given in units of $5.29 \times 10^{-11} \mathrm{~m}, 4.36 \times 10^{-18} \mathrm{~J}, 5.14 \times 10^{11} \mathrm{~V} / \mathrm{m}$, and $2.35 \times 10^{5} \mathrm{~T}$, respectively)

$$
H=\frac{1}{2} p^{2}-\frac{1}{r}+\frac{1}{2} B L_{z}+\frac{1}{8} B^{2}\left(x^{2}+y^{2}\right)+F x,
$$

with $L_{z}=x p_{y}-y p_{x}$. In the following we shall take advantage of a scaling property of the classical Hamiltonian enabling us to deal with only two independent variables, i.e., the scaled energy and the scaled field strength. The other variables have to be scaled, as well, so that the scaling transformations read

$$
\begin{gathered}
\tilde{E}=E B^{-2 / 3}, \tilde{F}=F B^{-4 / 3}, \\
\tilde{\boldsymbol{r}}=\boldsymbol{r} B^{2 / 3}, \tilde{\boldsymbol{p}}=\boldsymbol{p} B^{-1 / 3}, \tilde{t}=t B .
\end{gathered}
$$

Without further usage of the tilde sign the Hamiltonian has the same structure as in Eq. (1) when setting $B=1$. Defining $\gamma=(\boldsymbol{r}, \boldsymbol{p})^{T}$ we want to find periodic solutions of the Hamiltonian equations of motion

$$
\frac{\mathrm{d}}{\mathrm{d} t} \boldsymbol{\gamma}=\boldsymbol{J} \frac{\partial H}{\partial \gamma}, \text { with } \boldsymbol{J}=\left(\begin{array}{cc}
\mathbf{0} & \mathbf{1} \\
-\mathbf{1} & \mathbf{0}
\end{array}\right)
$$

The Stark saddle point is characterized by the fixedpoint condition $\dot{\gamma}=\mathbf{0}$, yielding its position $\boldsymbol{r}_{\mathrm{SP}}=$ $(-1 / \sqrt{F}, 0,0)^{T}$ and energy $E_{\mathrm{SP}}=-2 \sqrt{F}$. The stability of orbits is investigated using the stability matrix $\bar{M} . \bar{M}$ describes in a linear approximation the relative behavior of two trajetories $\gamma^{(1)}$ and $\gamma^{(2)}$ in time [39],

$$
\gamma^{(1)}(t)-\gamma^{(2)}(t)=\overline{\boldsymbol{M}}(t)\left(\gamma^{(1)}(0)-\gamma^{(2)}(0)\right) \text {. }
$$

and can be determined by its equation of motion

$$
\frac{\mathrm{d}}{\mathrm{d} t} \overline{\boldsymbol{M}}=\boldsymbol{J} \frac{\partial^{2} H}{\partial \gamma^{2}} \overline{\boldsymbol{M}}, \text { with } \boldsymbol{J}=\left(\begin{array}{cc}
\mathbf{0} & \mathbf{1} \\
\mathbf{- 1} & \mathbf{0}
\end{array}\right), \overline{\boldsymbol{M}}(0)=\mathbf{1}
$$

The eigenvalues of $\bar{M}(t)$ therefore indicate whether a variation of the initial conditions of a periodic orbit leads to exponential divergence or a trajectory remains in the vicinity of the periodic orbit for all times. In the case of a Hamiltonian system the energy conservation leads to two variational directions not affecting the system's behavior. The corresponding eigenvalues hence take on the value of 1 . Omitting of those directions one obtains the so-called monodromy matrix $\boldsymbol{M}$.
To prevent a divergence of the momentum $\boldsymbol{p}$ near the nucleus the Kustaanheimo-Stiefel regularization of coordinates [40, 41] is used,

$$
\begin{gathered}
\boldsymbol{r}=\boldsymbol{L}(\boldsymbol{U}) \boldsymbol{U}=\frac{1}{2}\left(\begin{array}{cccc}
U_{3} & -U_{4} & U_{1} & -U_{2} \\
U_{4} & U_{3} & U_{2} & U_{1} \\
U_{1} & U_{2} & -U_{3} & -U_{4} \\
U_{2} & -U_{1} & -U_{4} & U_{3}
\end{array}\right) \boldsymbol{U}, \\
\boldsymbol{p}=\frac{1}{r} \boldsymbol{L}(\boldsymbol{U}) \boldsymbol{P},
\end{gathered}
$$

together with a transformation of time,

$$
\mathrm{d} t=2 r \mathrm{~d} \tau
$$

The equations of motion (3) and (5) remain the same except for the replacements $\gamma \rightarrow \boldsymbol{\Gamma}=(\boldsymbol{U}, \boldsymbol{P})^{T}$ and $t \rightarrow$ $\tau$. The Hamiltonian then reads

$$
\begin{aligned}
H= & \frac{1}{2} P^{2}-\left[E-F\left(U_{1} U_{3}-U_{2} U_{4}\right)\right] U^{2} \\
+ & \frac{1}{2}\left[\left(U_{1} P_{2}-U_{2} P_{1}\right)\left(U_{3}^{2}+U_{4}^{2}\right)\right. \\
& \left.+\left(U_{3} P_{4}-U_{4} P_{3}\right)\left(U_{1}^{2}+U_{2}^{2}\right)\right] \\
+ & \frac{1}{8} U^{2}\left(U_{1}^{2}+U_{2}^{2}\right)\left(U_{3}^{2}+U_{4}^{2}\right)=2
\end{aligned}
$$

with $U^{2}=\sum_{i=1}^{4} U_{i}^{2}$. By integrating two further equations along with the Hamiltonian equations of motion one obtains the periods and actions of the orbits:

$$
\begin{aligned}
\frac{\mathrm{d}}{\mathrm{d} \tau} t=U^{2} & \\
\frac{\mathrm{d}}{\mathrm{d} \tau} S=P^{2}+\frac{1}{2}[ & \left(U_{1} P_{2}-U_{2} P_{1}\right)\left(U_{3}^{2}+U_{4}^{2}\right) \\
& \left.\quad+\left(U_{3} P_{4}-U_{4} P_{3}\right)\left(U_{1}^{2}+U_{2}^{2}\right)\right] .
\end{aligned}
$$

The transformation 6 is not bijective. For this reason the inversion,

$$
\boldsymbol{U}=\left(\begin{array}{c}
\sqrt{r+z} \cos \left(\frac{\varphi+\alpha}{2}\right) \\
\sqrt{r+z} \sin \left(\frac{\varphi+\alpha}{2}\right) \\
\sqrt{r-z} \cos \left(\frac{\varphi-\alpha}{2}\right) \\
\sqrt{r-z} \sin \left(\frac{\varphi-\alpha}{2}\right)
\end{array}\right)
$$

contains an additional parameter $\alpha$, which, without loss of generality, we will set to the constant value $\alpha=0$.

\section{B. Cusp bifurcation and exceptional points}

A cusp bifurcation, appearing in systems with at least two parameters $a$ and $b$, is described by the normal form 42

$$
\dot{x}=\frac{4}{27} x^{3}+a x+b
$$


We choose the factor in front of $x^{3}$ as $\frac{4}{27}$ instead of 1 to simplify the results. When calculating the fixed points of Eq. (11) one finds three real solutions for $a<0$ and $|b|<$ $(-a)^{3 / 2}$. The two boundary lines of this area are tangent bifurcation lines, along which two fixed points coincide. In the remaining parameter space only one real solution can be found. A specific attribute of the cusp bifurcation shows a similarity to exceptional points: Following one fixed point around the cusp point, which is located at $(a, b)=(0,0)$, along a circle

$$
(a, b)=(-r \cos \varphi,-r \sin \varphi), \text { with } \varphi \in[0,2 \pi)
$$

it can be transformed into another one. This phenomenon appears in the case of exceptional points, too. An exceptional point, first described by Kato [43, marks the coalescence of at least two resonances (or more precisely: eigenvalues and corresponding eigenvectors) of a complex Hamiltonian in an at least two-dimensional parameter space [44 47]. Encircling the exceptional point in parameter space the resonances permute. In the case of an EP2 two resonances interchange and the initial situation can be restored after two cycles (Fig. 1a and 1b). This can be described by the normal form of a tangent bifurcation

$$
\dot{x}=x^{2}-\mu,
$$

when choosing the parameter $\mu$ to be complex. The two complex fixed points can then be interchanged by encircling the exceptional point $\mu=0$ along the unit circle in the complex plane $\left(\mu=e^{i \varphi}, \varphi \in[0,2 \pi)\right)$. Similarly, the behavior of an EP $n$ can be described by the normal form

$$
\dot{x}=x^{n}-\mu .
$$

When encircling the exceptional point the $n$ fixed points permute and the initial situation is restored after $n$ cycles.

Depending on the path for the encircling the cusp bifurcation can show the behavior of an EP2 or an EP3: By choosing $b=0$ and $a=e^{i \varphi}$ one obtains an EP2-behavior. On the other hand an EP3-behavior is obtained by setting $a=0$ and $b=e^{i \varphi}$. This phenomenon was already observed in quantum mechanical calculations for dipolar Bose-Einstein condensates [46, 47]. The path of Eq. (12) with real parameters $a$ and $b$ gives rise to an ambiguity between both behaviors: If one traces two fixed points beyond a tangent bifurcation, two complex fixed points will appear. Since those two points coincide in the tangent bifurcation, it is not possible to relate one of the complex points to one of the real points, respectively. The permutation behavior cannot be determined, which is shown in Fig. 1 k and 1 d. This ambiguity exists only for real parameter values. If a small complex part is added to $a$ or $b$, an unambigious determination of the permutation behavior is possible.
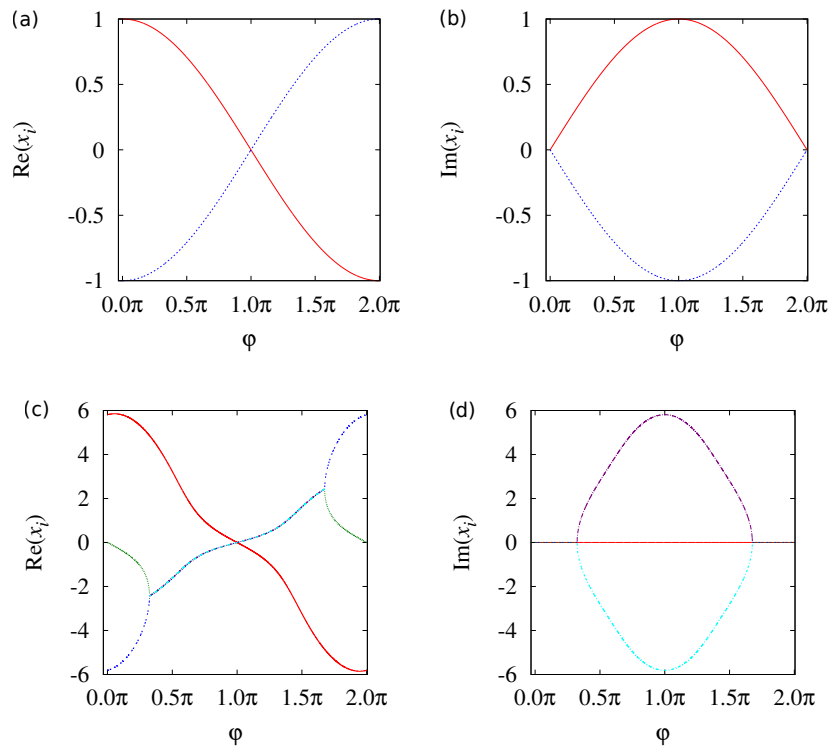

FIG. 1: (Color online) By encircling an EP2 an interchange of two resonances can be observed. The figures (a) and (b) show the real and imaginary part of the two fixed points of Eq. (13), depending on the angle $\varphi$ in $\mu=e^{i \varphi}$. (c), (d) When encircling the cusp point along $(a, b)=(-5 \cos \varphi,-5 \sin \varphi), \varphi \in$ $[0,2 \pi)$, one of the fixed points of Eq. 111 can be transformed into another one (marked by the red solid line starting in the upper left corner of figure (c)), without passing through a bifurcation. A second encircling passes both tangent bifurcations. Since the fixed points coincide in those bifurcations, it is not possible to determine the permutation behavior. An EP2- or an EP3-behavior can be observed accordingly. The angle $\varphi$ and the position $x$ are given in dimensionless units (see Eqs. 11) and (13).

\section{RESULTS OF CLASSICAL CALCULATIONS AND DISCUSSION}

Figure $2 \mathrm{a}$ shows the shape of the quasi-Penning resonances when following them up to higher energies at a fixed field strength. Since the name quasi-Penning was applied to almost elliptical orbits localized in the vicinity of the Stark saddle point and referred to the structural similarity between the equations of motion linearized around the saddle point and the stability conditions in a Penning trap we will now refer to them more generally as $B_{1}$. The differences to the case of energies slightly above $E_{\mathrm{SP}}$ are obvious: When increasing the energy the orbit becomes more and more heart-shaped. Figure $2 \mathrm{~b}$ shows in advance the shortest orbits in the $z=0$-plane, which are now investigated in detail.

In the following figures we indicate the stability of orbits by two upper indices added to the name of the orbit. The first one refers to the stability (s) or instability (u) perpendicular to the magnetic field. The second one indicates the stability parallel to the magnetic field. Regions of different stability are additionally dyed by colors, shadings and grayscales. The stability behavior of 

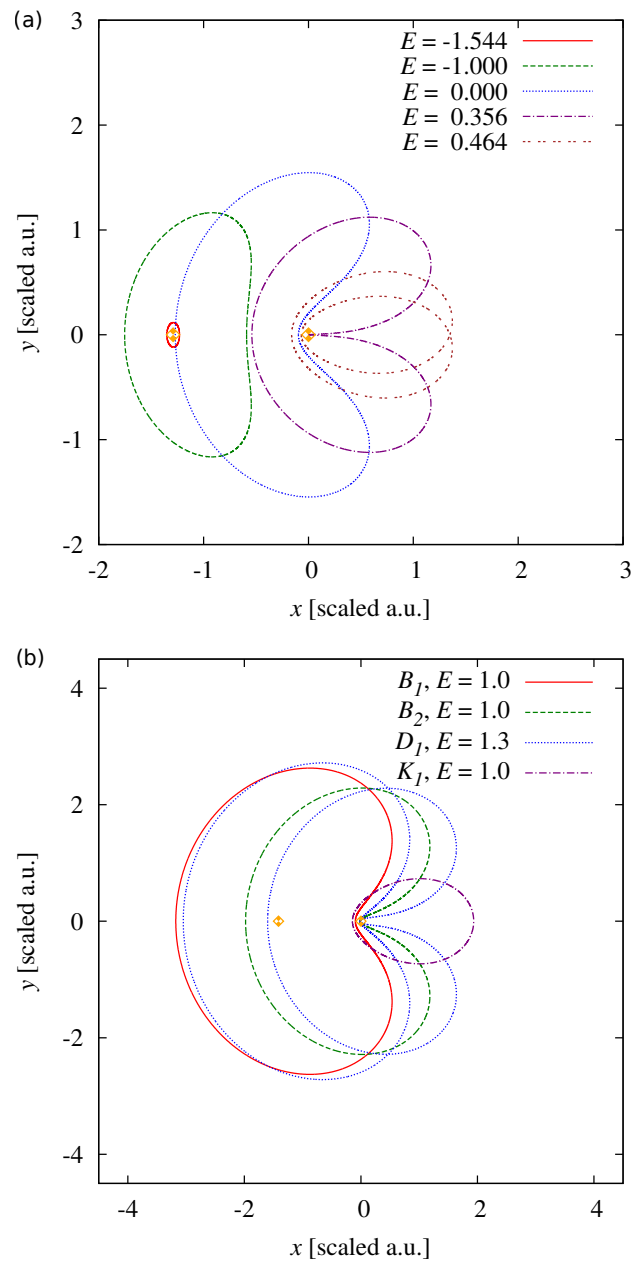

FIG. 2: (Color online) (a) Shape of the orbit $B_{1}$, evolving from the elliptically shaped quasi-Penning resonances, at different energies and a fixed value of $F=0.6$. The orbit is localized in the $z=0$-plane. The position of the Stark saddle point on the left hand side and the nucleus at the origin are marked by diamonds. At $E \approx 0.356$ the right point of intersection with the $x$-axis coincides with the nucleus. (b) Shape of the orbits $B_{1}, B_{2}, D_{1}$ and $K_{1}$ at $F=0.5$. The positions $x$ and $y$ are given in scaled atomic units (see Eq. (2)).

the orbits $B_{1}$ is shown in Figure 3 . After coming into existence they are at first stable parallel to the magnetic field, since the Stark saddle point is a local potential minimum in this direction. Towards higher energies they become completely unstable and may vanish in bifurcations. We note that above a specific field strength of $F_{x y, B K}=0.481$ the right point of intersection with the $x$-axis of the orbits $B_{1}$ coincides with the nucleus. This coincidence occurs along the dash dotted black line in Fig. 3a, which passes through the lower area of instability. Along this line the orbits $B_{1}$ are closed periodic orbits starting at and returning to the nucleus [48,50]. Afterwards a change of the shape takes place before the orbit vanishes in a pitchfork bifurcation with period-doubling along the right dashed line in Fig. 3 a.
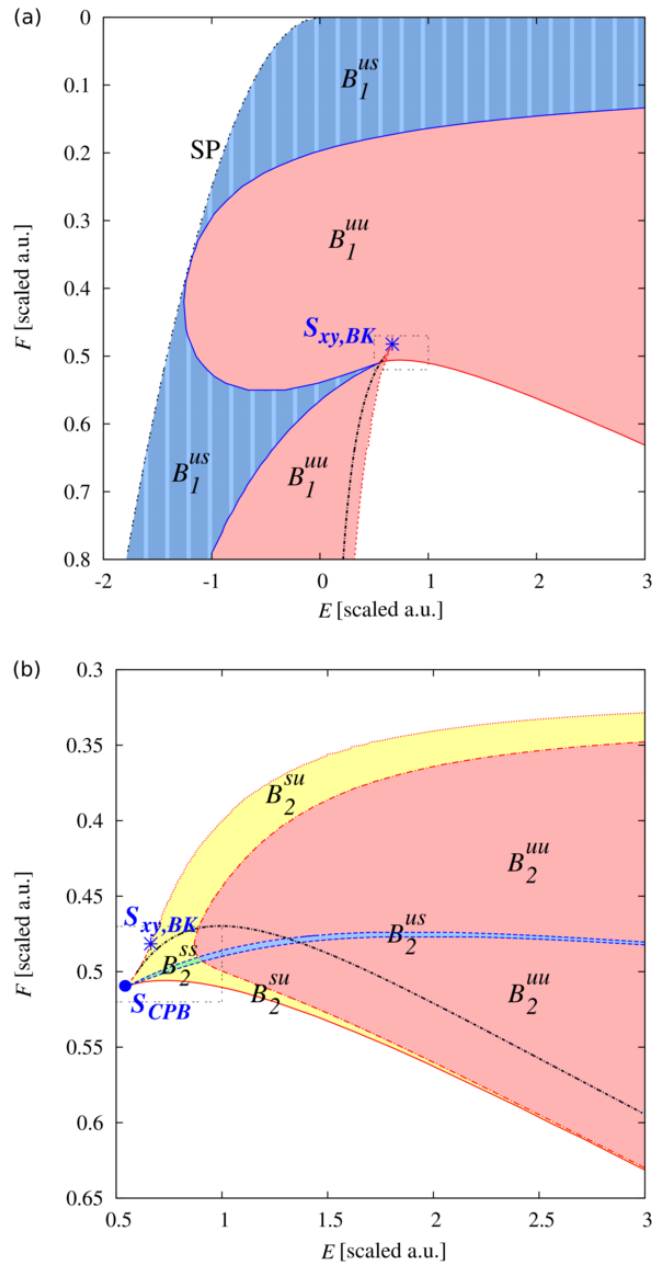

FIG. 3: (Color online) Stability of $B_{1}$ (a) and $B_{2}$ (b) in dependence on the scaled energy and the scaled field strength. Along the lines marking the borders of different stability regions bifurcations occur. The stability within those regions is displayed by two upper indices. The first one refers to the stability (s) or instability $(\mathrm{u})$ of the orbit in the direction perpendicular to the magnetic field, the second one to the stability in the direction parallel to the magnetic field. According to the different types of stability the regions are additionally dyed by colors, shadings and grayscales. One can clearly observe the stability apex for $B_{1}$ ending at $F \approx 0.5, E \approx 0.5$, i.e., a point in the $E-F$ space at which two border lines of the stability regions $B_{1}^{u s}-B_{1}^{u u}$ meet, which was first uncovered by Flöthmann [38]. The dashed line on the left hand side in (a) marks the energy of the saddle point (SP) as a function of the field strength. Specific points in parameter space are displayed by $\boldsymbol{S}_{i}=\left(F_{i}, E_{i}\right)$. The field strength $F$ and the energy $E$ are given in scaled atomic units (see Eq. (2)).

In crossed fields two orbits exist, which are localized in the $z=0$-plane and which change over to the Kepler orbits [9, 38, in the case of vanishing fields. We will refer to them as $K_{1}$ and $K_{2}$. The second orbit, $K_{2}$, distinguishing itself from the first one by its sense of rotation around the nucleus, is not involved in any of the bifurcations con- 

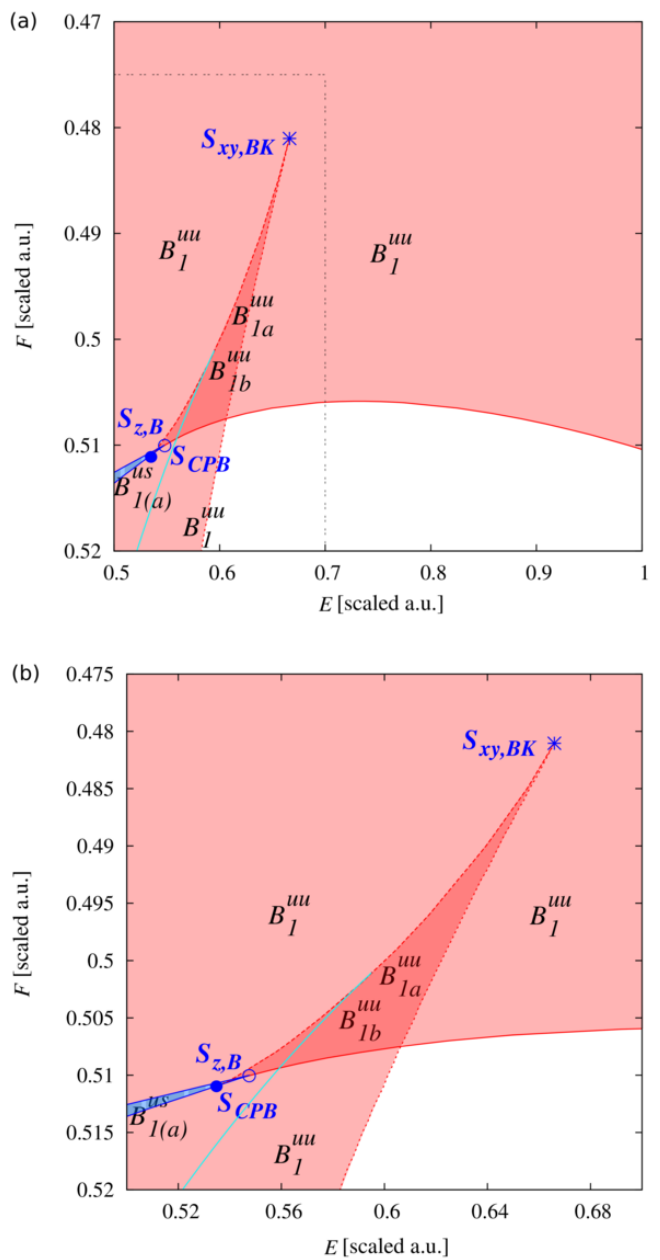

FIG. 4: (Color online) Stability of $B_{1}$ in dependence on the scaled energy and the scaled field strength. (a) is an enlargement of the rectangular area marked by dashed lines in Fig. 33. (b) is an enlargement of the equivalently marked area in (a). The different resolutions allow for a comparison with Fig. 6 and provide a better insight in the relevant region around $F \approx 0.5, E \approx 0.6$. In the dark red area two different versions of $B_{1}$ exist, which are indicated by $B_{1 a}$ and $B_{1 b}$. The field strength $F$ and the energy $E$ are given in scaled atomic units (see Eq. 2).

sidered and therefore not shown in Fig. 2p. The lastly mentioned dashed red line marks also a stability change perpendicular to the magnetic field of $K_{1}$, which is related to a bifurcation between $B_{1}$ and $K_{1}$. Beside the bifurcation with $K_{1}$ two other bifurcations can be observed for $B_{1}$ : At the stability change in $z$-direction pitchfork bifurcations with period-doubling occur, in which threedimensional orbits are created. Along the remaining solid black line at high energies separating the white area from the area of instability in Fig. 3 a a tangent bifurcation with $B_{2}$ occurs. This orbit has a similar shape as $B_{1}$, but exits only at high energies and field strengths (Fig. $3 \mathrm{~b}$ ). If one takes a closer look at the stability areas according to the $z$-direction one can find that they are ending in
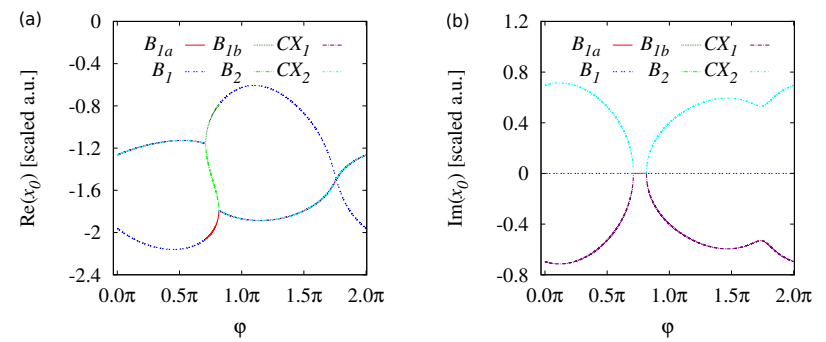

FIG. 5: (Color online) Real (a) and imaginary part (b) of one of the intersection points of the orbits with the $x$-axis when encircling the cusp point $\boldsymbol{S}_{\mathrm{CP} B}$. The angle $\varphi$ parameterizes the circle around $\boldsymbol{S}_{\mathrm{CP} B} . C X_{1}$ and $C X_{2}$ are the two complex orbits appearing. Just as in Fig. 11; and 1 1 it is not possible to determine the permutation behavior due to the coincidence of two orbits in a tangent bifurcation, respectively. The angle $\varphi$ and the values of $x_{0}$ are given in dimensionless and scaled atomic units, respectively (see Eq. (2)).

an apex not only for $B_{1}$ but also for $B_{2}$. Both apexes meet at the point $\boldsymbol{S}_{z, B}=\left(F_{z, B}, E_{z, B}\right)=(0.510,0.548)$ which is located on the bifurcation line between $B_{1}$ and $B_{2}$. Therefore we conclude that stability borders limiting the area of stability in $z$-direction for $B_{1}$ continue as the according stability borders of $B_{2}$.

Taking a closer look at this region of parameter space in Fig. $4 \mathrm{~b}$ a cusp bifurcation between orbits $B_{1}$ and $B_{2}$ is observed. As described in Sec. IIB two tangent bifurcation lines coincide in the cusp point - here $\boldsymbol{S}_{\mathrm{CP} B}=(0.511,0.535)$ - without continuation. Along both lines bifurcations between $B_{1}$ and $B_{2}$ occur. But while $B_{2}$ exists only in the area between both lines, $B_{1}$ exists in the complete external region and in the area between both lines twice. We will refer to those two different versions of $B_{1}$ as $B_{1 a}$ and $B_{1 b}$. The three orbits $B_{1 a}, B_{1 b}$ and $B_{2}$ correspond to the three fixed points of the cusp bifurcation in Sec. IIB. Starting from the lower continuous line at the right-hand side of $\boldsymbol{S}_{\mathrm{CP} B}$ we follow the orbit $B_{1 a}$ or $B_{1}$ anticlockwise around the cusp point and again in the darker marked area until it vanishes as $B_{1 b}$ in a tangent bifurcation along the dashed line between $\boldsymbol{S}_{\mathrm{CP} B}$ and $\boldsymbol{S}_{x y, B K}$. Therefore $B_{1 a}$ and $B_{1 b}$ can be converted into each other by encircling $\boldsymbol{S}_{\mathrm{CP} B}$. This behavior is the same as for the fixed points of Eq. (11). By allowing the coordinates and the time to become complex, the analytically continued orbits can be followed beyond the tangent bifurcations. A plot of one of the intersection points of the orbits with the $x$-axis vs. the angle $\varphi$, which parameterizes the circle around $\boldsymbol{S}_{\mathrm{CP} B}$, is shown in Fig. 5 .

The left tangent bifurcation line of $B_{1}$ and $B_{2}$ in Fig. 4 leading from $\boldsymbol{S}_{\mathrm{CPB}}$ to $\boldsymbol{S}_{x y, B K}$ coincides in $\boldsymbol{S}_{x y, B K}$ with the dashed pitchfork bifurcation line of $B_{1}$ and $K_{1}$ also ending in $\boldsymbol{S}_{x y, B K}$. It continues itself towards lower field strengths as a pitchfork bifurcation line between $B_{2}$ and $K_{1}$. This line marks the upper boundary of the region in which $B_{2}$ exists. Examinating the stability 

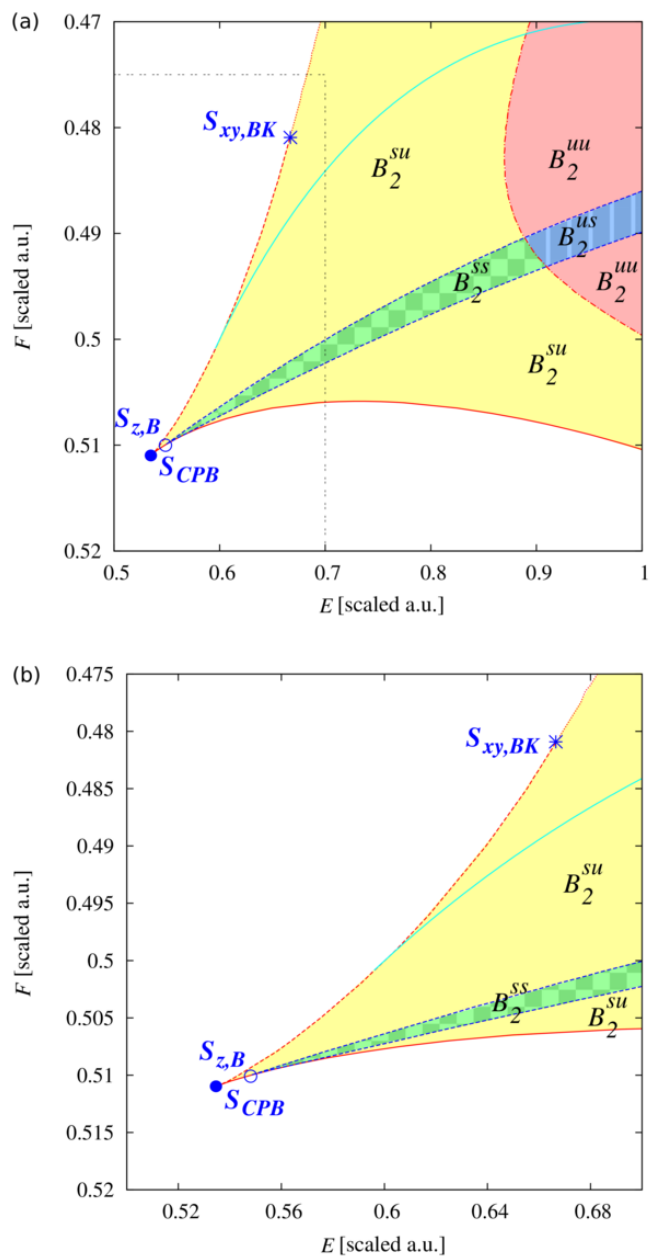

FIG. 6: (Color online) (a) Stability of $B_{2}$ in dependence on the scaled energy and the scaled field strength. The observed stability island is triangular in shape indicated by $B_{2}^{s s}$ and ends in $\boldsymbol{S}_{z, B}$. (b) is an enlargement of the equivalently marked area in (a). The field strength $F$ and the energy $E$ are given in scaled atomic units (see Eq. (2)).

behavior of $B_{2}$ one can notice another interesting phenomenon: The orbit becomes completely stable in a relatively large area of parameter space (green area marked $B_{2}^{s s}$ in Fig. 6). This is used as an opportunity to carry out a semiclassical quantizaton of $B_{2}$ and to search for signatures in exact quantum spectra in Sec. V

Finally one last orbit localized in the $z=0$-plane is worth mentioning. Along the dash dotted black line in Fig. $3 \mathrm{~b}$ describing a stability change of $B_{2}$ perpendicular to the magnetic field a pitchfork bifurcation with period-doubling occurs. The period-doubled orbit coming into being is named $D_{1}$ (compare Fig. 2). For $D_{1}$ both of the described phenomena can be found again! In Fig. 7b a very small region of complete stability localized at even higher energies than the stability island of $B_{2}$ is displayed. The cusp bifurcation occurs around the cusp point $\boldsymbol{S}_{\mathrm{CP} D}$, in which again two tangent bifurcation
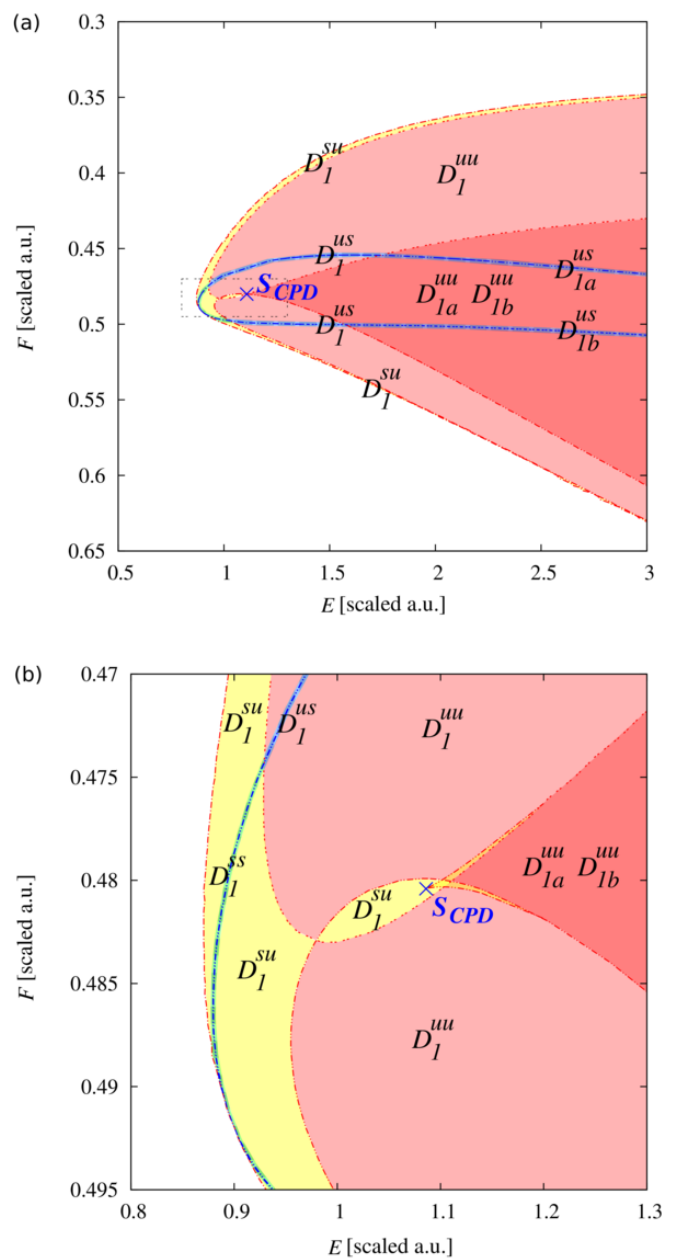

FIG. 7: (Color online) Stability of $D_{1}$ in dependence on the scaled energy and the scaled field strength. A very thin region marked $D_{1}^{s s}$ of complete stability can be observed. A cusp bifurcation occurs around the cusp point $\boldsymbol{S}_{\mathrm{CP} D}$. (b) is an enlargement of the equivalently marked area in (a). The field strength $F$ and the energy $E$ are given in scaled atomic units (see Eq. 22).

lines coincide without continuation. The darker marked areas denote the existence of two different versions of $D_{1}: D_{1 a}$ and $D_{1 b}$. We conclude that the reappearance of those phenomena indicates the possibility to find them for other orbits of even more complicated shape on-andoff-again.

\section{SEMICLASSICAL QUANTIZATION AND EXACT QUANTUM-MECHANICAL CALCULATIONS}

According to periodic orbit theory every classical periodic orbit causes a modulation in the density of states [51 60. Oscillating modulations can also be found in photoabsorption spectra or action spectra, respectively, 
and are according to Gutzwiller [52] and Miller [61] located at

$$
S-\sum_{i=1}^{2}\left(m_{i}+\frac{1}{2}\right) \varphi_{i}=2 \pi\left(n+\frac{\lambda}{4}\right)
$$

with the action $S$, the stability angles $\varphi_{i}=\arg \left(d_{i}\right)$, determined by the eigenvalues $d_{i}$ with $\left|d_{i}\right| \geq 1$ of the monodromy matrix, indicating the stability parallel $(\|)$ and perpendicular $(\perp)$ to the magnetic field, the Maslov index $\lambda$ and the quantum numbers $n, m_{1}, m_{2}$. Those quantum numbers count the number of quanta along $(n)$ and perpendicular $\left(m_{i}\right)$ to the periodic orbit. Stable periodic orbits cause modulations of the density of states, which can be described by $\delta$-function peaks, while unstable orbits cause broadened peaks. Those broadened peaks have the shape of Lorentzians in action spectra, i.e., when they are plotted against the action $S$. In the following we will use the term semiclassical half-width for the half-widths of those peaks according to energy. For each value of $n$ and each constant values of the field strengths the widths according to energy can be calculated as the difference between the two points, where

$$
S \pm \sum_{i=1}^{2} \ln \left|d_{i}\right|-\sum_{i=1}^{2}\left(m_{i}+\frac{1}{2}\right) \varphi_{i}=2 \pi\left(n+\frac{\lambda}{4}\right)
$$

is fulfilled [52]. Since we did not calculate the Maslov index of $B_{2}$, we assume in the following $\lambda=1$, which will supply the expected agreement between semiclassical and quantum-mechanical results.

The quantum-mechanical resonance spectra and wave functions are determined as described in 62]: The Schrödinger equation with the unscaled Hamiltonian (1) is rewritten in dilated semiparabolic coordinates [37, 63.

$$
\mu=\frac{1}{b} \sqrt{r+z}, \nu=\frac{1}{b} \sqrt{r-z}, \text { and } \varphi=\arctan \frac{y}{x} .
$$

Note that we cannot further use scaled variables in quantum mechanical calculations since the scaling is restricted to the classical dynamics [19, 38]. The parameter $b=|b| e^{i \vartheta / 2}$ introduces a complex delatation of the coordinates $\boldsymbol{r}$, which is necessary to determine resonances using the complex coordinate rotation method [64 66]. The Schrödinger equation then reads

$$
\begin{gathered}
{\left[\triangle_{\mu}+\triangle_{\nu}-\left(\mu^{2}+\nu^{2}\right)+4 b^{2}+b^{4} B\left(\mu^{2}+\nu^{2}\right) i \frac{\partial}{\partial \varphi}\right.} \\
\left.-\frac{1}{4} b^{8} B^{2} \mu^{2} \nu^{2}\left(\mu^{2}+\nu^{2}\right)-2 b^{6} F \mu \nu\left(\mu^{2}+\nu^{2}\right) \cos \varphi\right] \psi \\
=\left[\Lambda\left(\mu^{2}+\nu^{2}\right)\right] \psi,
\end{gathered}
$$

with the Laplacians

$$
\triangle_{\rho}=\frac{1}{\rho} \frac{\partial}{\partial \rho} \rho \frac{\partial}{\partial \rho}+\frac{1}{\rho^{2}} \frac{\partial^{2}}{\partial \varphi^{2}}, \rho \in\{\mu, \nu\}
$$
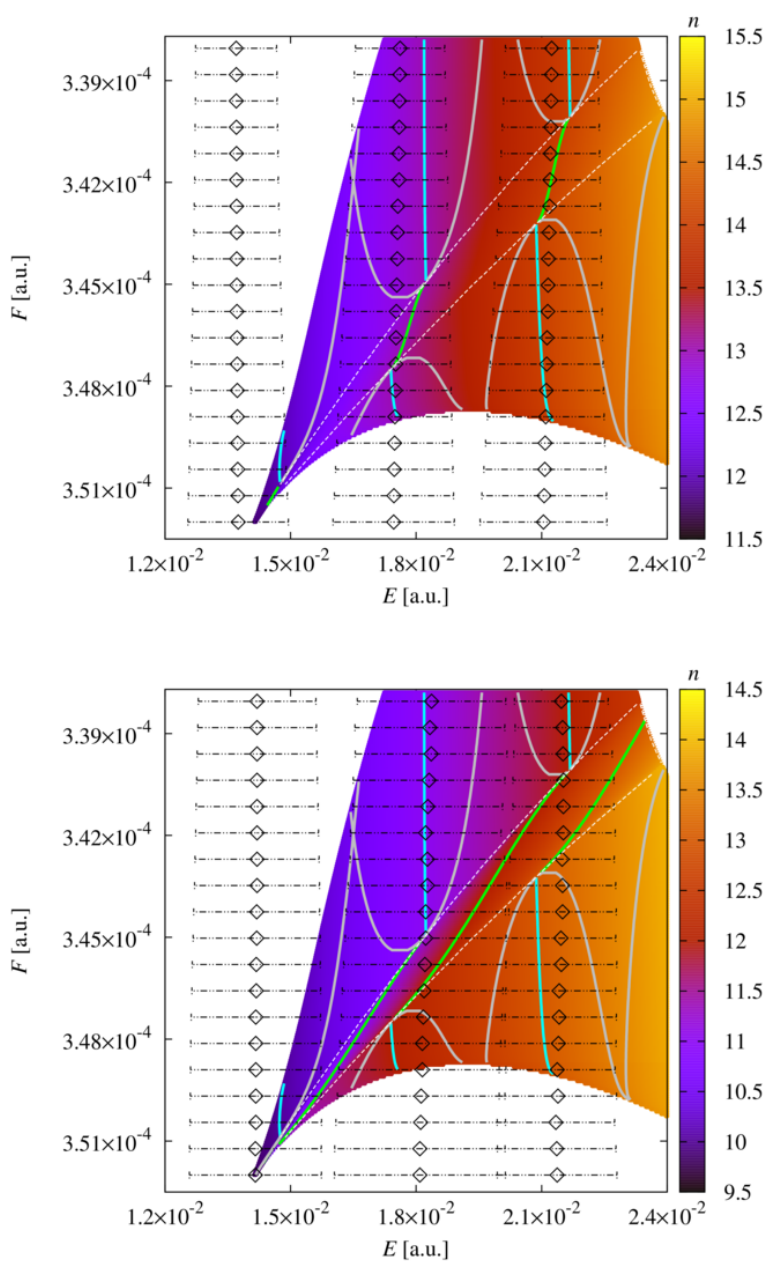

FIG. 8: (Color online) Comparison between quantummechanical and semiclassical results at $B=4.26 \times$ $10^{-3}(\hat{=} 1000 \mathrm{~T})$. For further information see text of Sec. V Best agreements are obtained for $\lambda=1$ and $m_{\perp}=0, m_{\|}=0$ in case of the first resonance series (a) and $m_{\perp}=0, m_{\|}=2$ in case of the second resonance series (b). The parameter range shown is almost the same as in Fig. 6a. All values are given in atomic units.

and generalized complex eigenvalues $\Lambda=-\left(1+2 b^{4} E\right)$, related to the complex energies $E$ of resonances, the real parts of which representing their energies and the imaginary parts their widths $\Gamma=-2 \operatorname{Im}(E)$. To calculate resonances a matrix representation of the Schrödinger equation $(18)$ is diagonalized. We use the adequate complete basis

$$
\left|n_{\mu}, n_{\nu}, m\right\rangle=\left|n_{\mu}, m\right\rangle \otimes\left|n_{\nu}, m\right\rangle
$$

with the eigenstates $\left|n_{\rho}, m\right\rangle$ of the two-dimensional harmonic oscillator. The position space representation reads

$$
\psi_{n_{\mu} n_{\nu} m}(\mu, \nu, \varphi)=\left\langle\mu, \nu, \varphi \mid n_{\mu}, n_{\nu}, m\right\rangle
$$




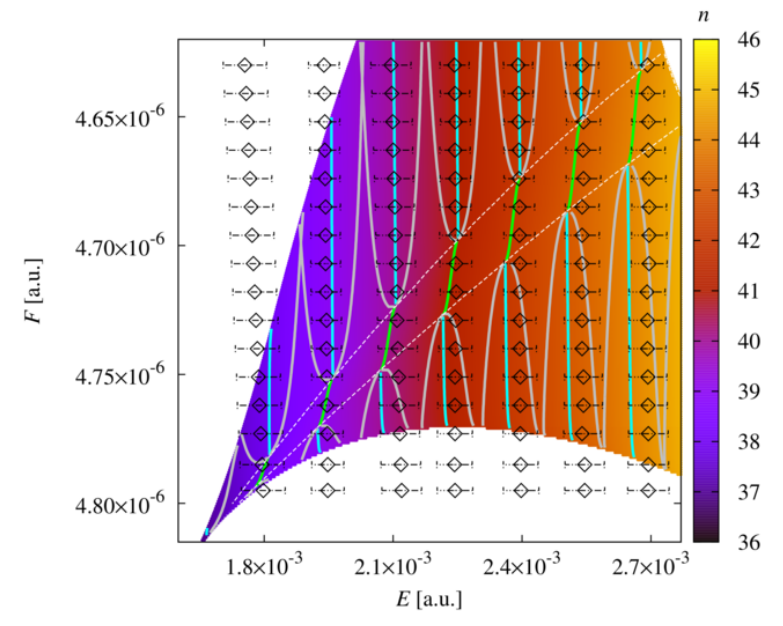

FIG. 9: (Color online) Comparison between semiclassical and quantum-mechanical results for the first resonance series at $B=1.70 \times 10^{-4}(\hat{=} 40 \mathrm{~T})$. The resonance widths are evidently smaller but still do not vanish within the stability island, which is marked by dashed lines. For further information see text of Sec. V] All values are given in atomic units.

$$
\begin{gathered}
=\sqrt{\frac{\left[\left(n_{\mu}-|m|\right) / 2\right] !\left[\left(n_{\nu}-|m|\right) / 2\right] !}{\left[\left(n_{\mu}+|m|\right) / 2\right] !\left[\left(n_{\nu}+|m|\right) / 2\right] !}} \\
\quad \times \sqrt{\frac{2}{\pi} f_{n_{\mu} m}(\mu) f_{n_{\nu} m}(\nu) e^{i m \varphi}}
\end{gathered}
$$

with

$$
f_{n m}(\rho)=e^{-\rho^{2} / 2} \rho^{|m|} \mathcal{L}_{(n-|m|) / 2}^{|m|}\left(\rho^{2}\right)
$$

and the associated Laguerre polynomials $\mathcal{L}_{k}^{\alpha}(x)$. In our numerical calculations the maximum number of states used is limited by the condition $n_{\mu}+n_{\nu} \leq 60$ due to the required computer memory. Since $\mu$ and $\nu$ are complex coordinates, all non-intrinsically complex parts must remain unconjugated in case of a complex conjugation [66, 67]. This means that $\psi_{n_{\mu} n_{\nu} m}^{*}$ is equal to $\psi_{n_{\mu} n_{\nu} m}$ except for the term $e^{i m \varphi}$ which is replaced with $e^{-i m \varphi}$. The eigenstates

$$
\begin{aligned}
& \Psi_{i}(\mu, \nu, \varphi)=\sum_{n_{\mu}, n_{\nu}, m} c_{i n_{\mu}, n_{\nu}, m} \psi_{n_{\mu} n_{\nu} m}(\mu, \nu, \varphi) \\
& \Psi_{i}^{*}(\mu, \nu, \varphi)=\sum_{n_{\mu}, n_{\nu}, m} c_{i n_{\mu}, n_{\nu}, m} \psi_{n_{\mu} n_{\nu} m}^{*}(\mu, \nu, \varphi),
\end{aligned}
$$

obtained as a result of the matrix diagonalization, are normalized according to

$$
\int \mathrm{d}^{3} \boldsymbol{r} \Psi_{i}^{*}(\mu, \nu, \varphi) \Psi_{j}(\mu, \nu, \varphi)
$$
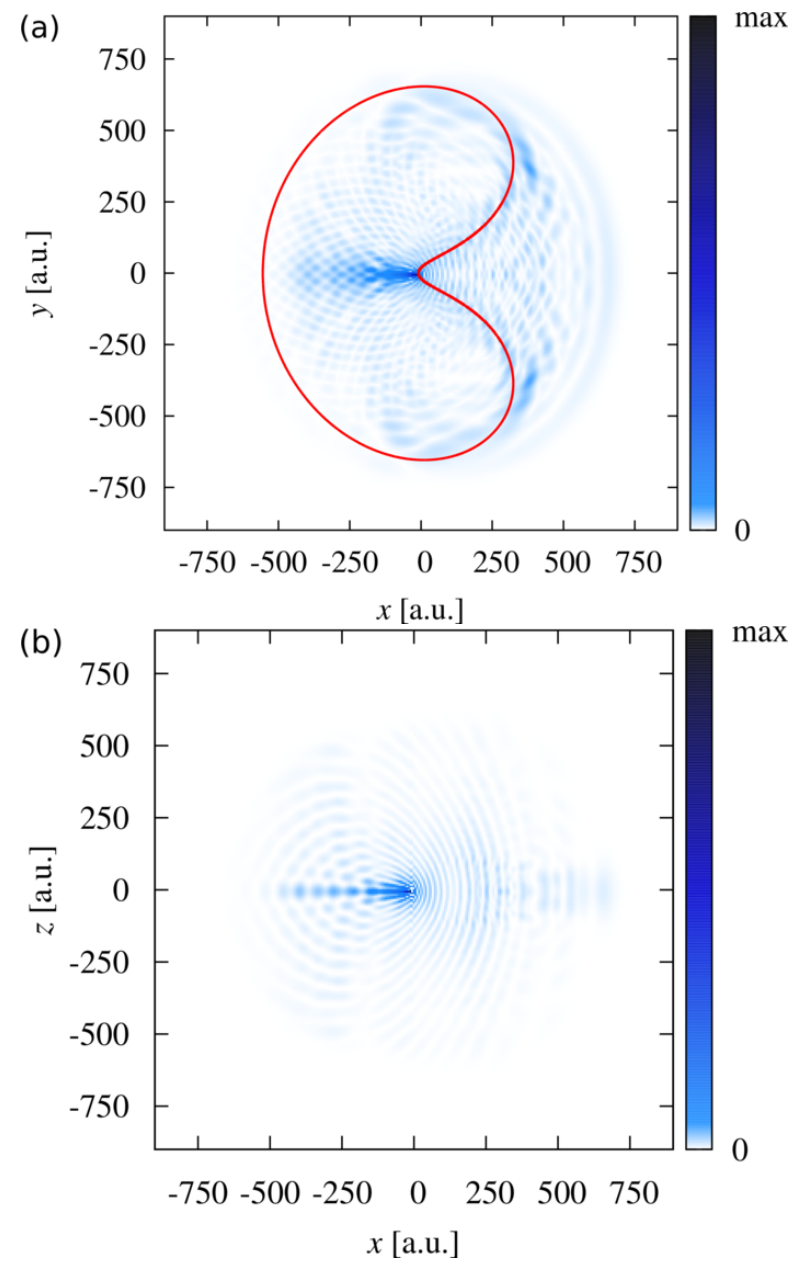

FIG. 10: (Color online) Probability density $\varrho(\boldsymbol{r})$ of a resonance of the first series at $E=1.95 \times 10^{-3}-4.14 \times 10^{-5} i$, $F=4.76 \times 10^{-6}$ and $B=1.70 \times 10^{-4}$. One can observe an agreement with the course of the classical orbit (continuous line) in (a) and the restriction to the $z=0$-plane in (b). All values are given in atomic units.

$$
=b^{6} \int_{0}^{\infty} \mathrm{d} \mu \int_{0}^{\infty} \mathrm{d} \nu \int_{0}^{2 \pi} \mathrm{d} \varphi \mu \nu\left(\mu^{2}+\nu^{2}\right) \Psi_{i}^{*} \Psi_{j}=\delta_{i j} .
$$

Due to the restriction of the complex conjugation to the intrinsically complex parts the expression $\Psi_{j}^{*} \Psi_{j}$ is not a real quantity. The probability density is obtained as $\varrho=\left|\Psi_{j}^{*} \Psi_{j}\right|$ [68] instead. Finally Husimi distributions can be calculated, allowing a comparison between the classical torus structure existing around the periodic orbits and quantum-Poincaré sections 69. To prevent a divergence of the momenta we use regularized coordinates for the Husimi distributions, too. We will show that the probability density of the observed resonances is mainly limited to the $z=0$-plane. For that reason the calculations can be restricted to the $x$ - and $y$ - or $U_{1}$ - and $U_{2}$-coordinate, respectively (cf. Eq. 6a and (10). By 

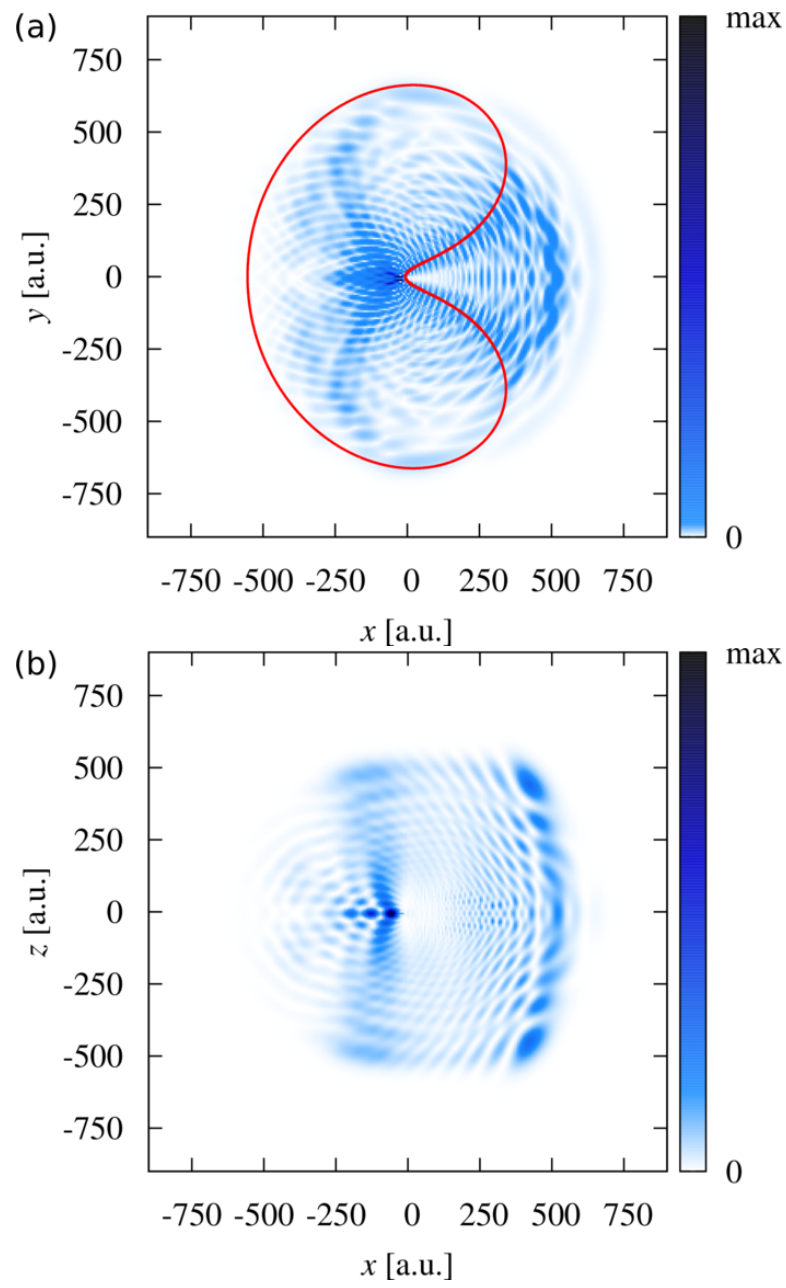

FIG. 11: (Color online) Probability density $\varrho(\boldsymbol{r})$ of a resonance of the second series at $E=2.14 \times 10^{-3}-5.87 \times 10^{-5} i$, $F=4.73 \times 10^{-6}$ and $B=1.70 \times 10^{-4}$. The differences to the classical orbit (continuous line) are significantly larger. All values are given in atomic units.

setting $z=0$ one obtains

$$
\left(\begin{array}{l}
x \\
y
\end{array}\right)=\left(\begin{array}{c}
U_{1}^{2}-U_{2}^{2} \\
2 U_{1} U_{2}
\end{array}\right)
$$

which is a bijection assuring $\alpha=0$ as long as $U_{2} \geq 0$ holds. The Husimi distribution 7073 then reads

$$
\begin{gathered}
P_{H}(\boldsymbol{U}, \boldsymbol{P})=\left(\int \mathrm{d}^{2} \boldsymbol{\Xi} 4|\boldsymbol{\Xi}|^{2} \Psi(\boldsymbol{\Xi}) G(\boldsymbol{\Xi}, \boldsymbol{U}, \boldsymbol{P})\right) \\
\quad \times\left(\int \mathrm{d}^{2} \boldsymbol{\Xi} 4|\boldsymbol{\Xi}|^{2} \Psi^{*}(\boldsymbol{\Xi}) G^{*}(\boldsymbol{\Xi}, \boldsymbol{U}, \boldsymbol{P})\right),
\end{gathered}
$$

with a Gaussian of minimum uncertainity $(\Delta U \Delta P=1 / 2)$

$$
G(\boldsymbol{\Xi}, \boldsymbol{U}, \boldsymbol{P})=\frac{1}{\left(\sigma^{2} \pi\right)^{1 / 2}} e^{-\frac{1}{2 \sigma^{2}}(\boldsymbol{\Xi}-\boldsymbol{U})^{2}-i(\boldsymbol{P}+\boldsymbol{A}(\boldsymbol{U})) \boldsymbol{\Xi}},
$$

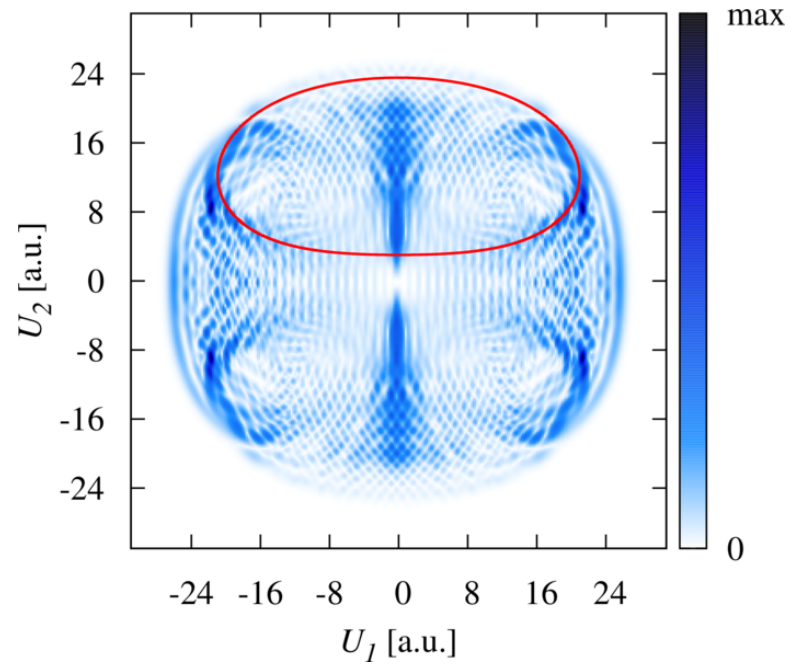

FIG. 12: (Color online) Probability density $\left|4 U^{2} \Psi^{*}(\boldsymbol{U}) \Psi(\boldsymbol{U})\right|$ of the resonance of Fig. 10 in regularized coordinates. Without the limitation of $U_{2} \geq 0$ the probability density shows a second symmetry relative to the line $U_{2}=0$. The lower part $\left(U_{2} \leq 0\right)$ corresponds to $\alpha=2 \pi$ and is not included in the calculations of the quantum-Poincaré sections. The classical orbit intersects the $U_{1}=0$-line twice but with a different sign of the velocity $V_{1}$ in $U_{1}$-direction. All values are given in atomic units.

the vector potential

$$
\boldsymbol{A}=\boldsymbol{P}+\frac{1}{2} B\left(U_{1}^{2}+U_{2}^{2}\right)\left(\begin{array}{c}
-U_{2} \\
U_{1}
\end{array}\right)
$$

and an appropriate adaptable squeezing parameter $\sigma=$ $\sqrt{\Delta U / \Delta P}$. To determine quantum-Poincaré sections $U_{1}$ is set to the constant value $U_{1}=0$. The conjugate momentum $P_{1}$ is then calculated according to Eq. (7).

\section{RESULTS OF QUANTUM-MECHANICAL CALCULATIONS AND DISCUSSION}

Since the classical calculations have been carried out for scaled energy values and scaled field strengths one of the three parameters $E, F$ and $B$ can now be chosen arbitrarily in quantum-mechanical calculations. In the following we will set $B$ to fixed values while the other parameters are calculated according to Eq. (2) and the location of the stability island. Figure 8 shows results at $B=1000 \mathrm{~T}$. The colorbar (grayscales) of this figure shows the quantum numbers $n$ obtained by semiclassical quantization of $B_{2}$ according to Eq. (15). The stability island is marked by dashed lines. Green (solid) lines within the stability island and blue (solid) lines outside of it display positions where integer values of $n$ are obtained. White (solid) lines of parabolic shape outside the stability island display the semiclassical half-widths calculated by Eq. (16). For each constant value of $F$ the semiclassical half-width (according to energy) of a semiclassical state 

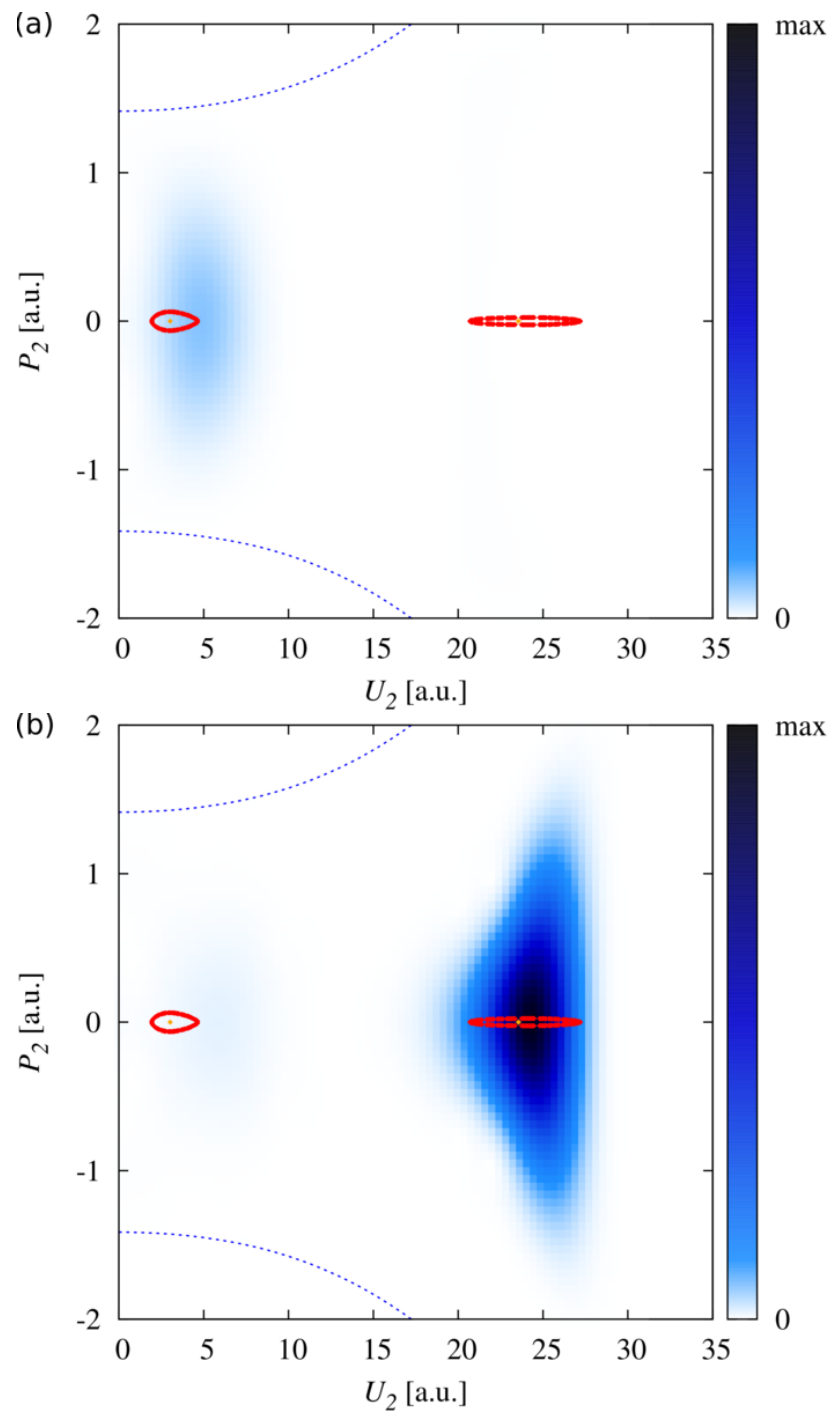

FIG. 13: (Color online) Comparison between quantumPoincaré sections (shown by colorbar (grayscales)) and classical torus structure. The dashed lines display the borders of the classically permitted region. Since the Hamiltonian is a quadratic function of the momentum, there are two possibilities to choose $P_{1}$ when calculating the section. Those both solutions differ from each other in the sign of the velocity $V_{1}$ in $U_{1}$-direction. Therefore agreements with only one part of the torus structure can be achieved at any time. The colorbar is chosen equal to compare both results. We set $\sigma$ to the constant value $\sigma=2$. All values are given in atomic units.

with quantum number $n$ can be read out as the sum of the distances between the blue (solid) line for this value of $n$ and the neighboring white (solid) lines. The real parts of the quantum-mechanical resonances (calculated only at discrete field strengths) are displayed by diamonds, the imaginary parts by error bars.

Two series of resonances were found, which can be traced down to lower energies. It is supposed that this retracing will end in the resonances described in 62 . The contradictory fact that the resonances can also be found outside the region where the classical orbits $B_{2}$ exist is an indication that a simple semiclassical quantization including solely $B_{2}$ may be insufficient (cf. also Sec. VI). Semiclassical quantization normally includes all orbits appearing at a specific point in parameter space [51. However, we assume that the resonances found within the area of $B_{2}$ are closely related to those orbits.

The energetic distance between two resonances according to the real part of the energy is almost the same within both series while the widths differ strongly between the two series. Assuming that the resonances of smaller widths (first series) represent a quantummechanical ground state and the other ones (second series) an exited state, semiclassical calculations are carried out to the quantum numbers $m_{\perp}=0, m_{\|}=0$ and $m_{\perp}=0, m_{\|}=2$, respectively. Since we consider only states of even parity in our quantum-mechanical calculations, the state with $m_{\perp}=0, m_{\|}=2$ must be the first excited state appearing in the spectra. As concerns the positions of the resonances a very good agreement between semiclassical and quantum-mechanical results is achieved outside the stability island. However, those quantum numbers lead to a switching of the semiclassical results from one resonance to another. Using different quantum numbers an even worse agreement outside the stability island is obtained. We therefore conclude that $m_{\perp}=0$ and $m_{\|}=2$ are correct and that the quantum mechanical resonances do not show the switching behavior since they are not able to resolve the semiclassical structure in this region, which we will explain in the following. On the other hand this switching can be seen as a further hint that a simple semiclassical quantization of $B_{2}$ is insufficient and that all the other orbits have to be considered as well.

As can be seen from Fig. 8, a good agreement according to the widths of the resonances is achieved only far away from the stability island. The expected decline of resonance widths within the stability island, proving the existence of quantum-mechanical bound states related to the classical stable orbits $B_{2}$, is not observed and will only show up at lower magnetic field strengths. When calculating Poincaré sections of the classical torus structure existing around the periodic orbit one uncovers that this structure is very small, too small to be resolved in quantum-mechnical calculations at $B=1000 \mathrm{~T}$.

Due to the expansion of this structure with a decreasing value of $B$ (note that $\boldsymbol{r}=\tilde{\boldsymbol{r}} B^{-2 / 3}$ ) one expects a better resolution at lower field strengths. The increasing quantum number of $n$ makes it necessary to increase the number of basis functions used in order to ensure a convergence of the quantum-mechanical calculations. Within the limitation of $n_{\mu}+n_{\nu} \leq 60$ it is possible to follow the resonances down to $B=40 \mathrm{~T}$. Figure 9 shows that even at this field strength the resonance widths do not vanish within the stability island. The probability densities (Fig. 10 and 11) exhibit instead a partially good agreement with the course of the classical orbit. Differences can be explained by the fact that the calculated 
wave function is only a snapshot while the resonance itself is a time evolving and finally decaying state. The higher quantum mechanical probability density along the negative $x$ axis possibly indicates the decay of the resonance since - from a classical point of view - all trajectories of the electron describing the ionization of the hydrogen atom have to pass the vicinity of the Stark saddle point 62]. This higher probability density then shows up again along the $U_{2}$ axis in Fig. 12 (and will finally be seen as a displacement between classical and quantum mechanical structure in Fig. 13 a. It can be observed that the larger values of the probability density are taken on in the $z=0$-plane when regarding the first resonance series. In case of the second resonance series the extension in the $z$-direction is much larger.

The calculated quantum-Poincaré sections are compared with the classical ones in Fig. 13. As expected, the torus structure is, especially in direction of the momentum $P_{2}$, much smaller than the quantum-mechanical structure.

Finally we want to estimate at which field strengths a solution of the classical structure may be possible. Since the classical orbit is localized in the $z=0$-plane we regard the extension of the torus structure around the orbit in a $z-p_{z}$-Poincaré section as its classical uncertainity (note that we change back to non-regularized coordinates in this place). Assuming that the quantum-mechanically determined uncertainity product $\Delta z \Delta p_{z}=1.59$ for one resonance of the first series does not change to a great extent with the value of $B$, since it is already sufficiently close to the critical value of $\frac{1}{2}$, we obtain a coincidence not until $B \approx 90 \mathrm{mT}$ ! This field strength is convenient for an experimental proof of the orbits $B_{2}$ in photoabsorption spectra. As it has been described, one of the intersection points of the classical orbits with the $x$-axis is very close to the nucleus within the relevant region around $F \approx 0.5, E \approx 0.6$. An exisiting overlap of the corresponding resonances and the lowest states (1s or $2 p$ ) of the hydrogen atom allows therefore an experimental excitation of the hydrogen atom into those resonance states. According to closed orbit theory [48, 50] and periodic orbit theory 5260 the classical periodic orbits then become noticeable in photoabsorption spectra by oscillating terms of the form $A \sin \left(2 \pi \tilde{S} B^{-1 / 3}+\varphi\right)$, in which the amplitudes depend on the stability of the orbits as well as the process of excitation. For the purpose of an experimental proof the data of an classical orbit located in the center of the stability island is given in scaled atomic units: $\tilde{F}=0.4985, \tilde{E}=0.75, \tilde{S}=14.7843$. The conversion to unscaled units at the chosen magnetic field strength is obtained by Eq. $(2)$ and $\tilde{S}=S B^{1 / 3}$.

\section{SUMMARY AND CONCLUSION}

Following the quasi-Penning resonances up to high energies and field strengths we could resolve their complete bifurcation behavior. We found out that only $K_{1}, B_{2}$ and three-dimensional orbits are involved in the bifurcations of $B_{1}$. It was shown that several phenomena appear in the region of $F \approx 0.5, E \approx 0.6$ including a cusp bifurcation between $B_{1}$ and $B_{2}$ as well as the appearance of a stability island for $B_{2}$. The reappearance of those phenomena when analysing the stability and bifurcation behavior of $D_{1}$ could then be interpreted as a possibility to find them on-and-off-again for orbits of even more complicated shape. The puzzle of the $z$-stability region of $B_{1}$ ending in an apex in parameter space could be resolved by a closer examination of the stability of $B_{2}$. The results show a coincidence of two stability apexes at $\boldsymbol{S}_{z, B}$, which indicates the continuation of the stability borders limiting the areas of stability in $z$-direction from one of those orbits to the other one.

Semiclassical quantizations of $B_{2}$ showed agreements with resonances found in exact quantum spectra. Due to further agreements between quantum-mechanical probability densities and classical orbits as well as between quantum-Poincaré sections and classical torus structure we have to conclude that signatures of the stable orbit $B_{2}$ in exact quantum spectra have been found. Owing to limited computer memory and power it is not yet possible to reach regions of several millitesla in order to find out whether the widths of the detected resonances will disappear within the stability island or not. Nevertheless we think that an experimental proof of $B_{2}$ in photoabsorption spectra at the estimated field strengths will eventually be possible.

Finally, since the widths of the observed resonances do not vanish within the stability island of $B_{2}$ and since the stability island is very close to the bifurcation lines with $B_{1 a}$ and $B_{1 b}$, we think that the orbits $B_{2}$ cannot be treated as isolated ones in a semiclassical quantization. It would be therefore preferable to perform a uniform semiclassical quantization [74 76] of the observed cusp bifurcation.

\section{Acknowledgments}

We thank Eugen Flöthmann for stimulating discussions.
[1] H. Rottke, H. Zacharias, and K. Welge, in Laser techniques for extreme ultraviolet spectroscopy, edited by T. McIlrath and R. Freeman (New York, 1982), vol. 90 of AIP Conf. Proc., pp. 402-421.

[2] R. Wallenstein, Opt. Comm. 33, 119 (1980).

[3] G. Wiebusch, J. Main, K. Krüger, H. Rottke, A. Holle, 
and K. Welge, Phys. Rev. Lett. 62, 2821 (1989).

[4] G. Raithel and H. Walther, Phys. Rev. A 49, 1646 (1994).

[5] G. Raithel, M. Fauth, and H. Walther, Phys. Rev. A 44, 1898 (1991).

[6] J. von Milczewski, G. Diercksen, and T. Uzer, Phys. Rev. Lett. 76, 2890 (1996).

[7] R. Cushman and D. Sadovskií, Physica D 142, 166 (2000).

[8] J. Main and G. Wunner, Phys. Rev. Lett. 69, 586 (1992).

[9] E. Flöthmann, J. Main, and K. Welge, J. Phys. B: At. Mol. Opt. Phys. 27, 2821 (1994).

[10] E. Flöthmann and K. Welge, Phys. Rev. A 54, 1884 (1996).

[11] T. Uzer and D. Farrelly, Phys. Rev. A 52, R2501 (1995).

[12] C. Neumann, R. Ubert, S. Freund, E. Flöthmann, B. Sheehy, K. Welge, M. Haggerty, and J. Delos, Phys. Rev. Lett. 78, 4705 (1997).

[13] J. von Milczewski, D. Farrelly, and T. Uzer, Phys. Rev. A 56, 657 (1997).

[14] D. Wang and J. Delos, Phys. Rev. A 63, 043409 (2001).

[15] G. Stania and H. Walther, Phys. Rev. Lett. 95, 194101 (2005).

[16] E. Lee, A. Puzder, M. Chou, T. Uzer, and D. Farrelly, Phys. Rev. B 57, 12281 (1998).

[17] M. Dignam and J. Sipe, Phys. Rev. B 45, 6819 (1992).

[18] M. Robnik, J. Math. Phys. 14, 3195 (1981).

[19] H. Harada and H. Hasegawa, J. Phys. A 16, L259 (1983).

[20] D. Delande and J. Gay, Phys. Rev. Lett. 57, 2006 (1986).

[21] D. Wintgen and H. Friedrich, Phys. Rev. Lett. 57, 571 (1986).

[22] G. Wunner, U. Woelk, I. Zech, G. Zeller, T. Ertl, F. Geyer, W. Schweizer, and H. Ruder, Phys. Rev. Lett. 57, 3261 (1986).

[23] A. Holle, J. Main, G. Wiebusch, H. Rottke, and K. Welge, Phys. Rev. Lett. 61, 161 (1988).

[24] T. van der Veldt, W. Vassen, and W. Hogervorst, Europhys. Lett 21, 903 (1993).

[25] J. Main, G. Wiebusch, K. Welge, J. Shaw, and J. Delos, Phys. Rev. A 49, 847 (1994).

[26] J. Yeazell, G. Raithel, L. Marmet, H. Held, and H. Walther, Phys. Rev. Lett. 70, 2884 (1993).

[27] C. Clark, E. Korevaar, and M. Littmann, Phys. Rev. Lett. 54, 320 (1985).

[28] S. Bhattacharya and A. Rau, Phys. Rev. A 26, 2315 (1982).

[29] A. Rau and L. Zhang, Phys. Rev. A 42, 6342 (1990).

[30] M. Fauth, H. Walther, and E. Werner, Z. Phys. D 7, 293 (1987).

[31] G. Raithel, M. Fauth, and H. Walther, Phys. Rev. A 47, 419 (1993).

[32] J. Gay, L. Pendrill, and B. Cagnac, Phys. Lett. A 72, 315 (1979).

[33] T. Uzer, C. Jaffé, J. Palacián, P. Yanguas, and S. Wiggins, Nonlinearity 15, 957 (2002).

[34] C. Jaffé, D. Farrelly, and T. Uzer, Phys. Rev. Lett. 84, $610(2000)$.

[35] C. Jaffé, D. Farrelly, and T. Uzer, Phys. Rev. A 60, 3833 (1999).

[36] S. Wiggins, L. Wiesenfeld, C. Jaffé, and T. Uzer, Phys. Rev. Lett. 86, 5478 (2001).

[37] H. Cartarius, J. Main, and G. Wunner, Phys. Rev. A 79,
033412 (2009).

[38] E. Flöthmann, Ph.D. thesis, Universität Bielefeld (1994).

[39] H. Meyer, J. Chem. Phys. 84, 3147 (1986).

[40] P. Kustaanheimo and E. Stiefel, J. Reine Angew. Math. 218, 204 (1965).

[41] E. Stiefel and G. Scheifele, Linear and regular celestial mechnics, vol. 174 of Grundlehren der mathematischen Wissenschaften (Springer, Berlin, 1971).

[42] T. Poston and I. Stewart, Catastrophe Theory and its Applications (Pitman Publishing Limited, London, 1978).

[43] T. Kato, Perturbation Theory of Linear Operators (Springer, Berlin, 1966).

[44] W. Heiss and A. Sannino, J. Phys. A: Math. Gen. 23, 1167 (1990).

[45] W. Heiss, Eur. Phys. J. 7, 1 (1999).

[46] G. Demange and E.-M. Graefe, J. Phys. A: Math. Theor. 45, 025303 (2012).

[47] R. Gutöhrlein, J. Main, H. Cartarius, and G. Wunner, J. Phys. A: Math. Theor. 46, 305001 (2013).

[48] M. Du and J. Delos, Phys. Rev. Lett. 58, 1731 (1987).

[49] J. Delos and M. Du, IEEE J. Quant. Electron. 24, 1445 (1988).

[50] E. Bogomol'nyi, JETP Lett. 47, 526 (1988).

[51] M. Brack and R. Bhaduri, Semiclassical Physics (Westview Press, Boulder, Colorado, 2003).

[52] M. Gutzwiller, J. Math. Phys. 12, 343 (1971).

[53] R. Balian and C. Bloch, Ann. Phys. 60, 401 (1970).

[54] R. Balian and C. Bloch, Ann. Phys. 64, 271 (1971).

[55] R. Balian and C. Bloch, Ann. Phys. 69, 76 (1972).

[56] R. Balian and C. Bloch, Ann. Phys. 85, 514 (1974).

[57] M. Gutzwiller, J. Math. Phys. 8, 1979 (1967).

[58] M. Gutzwiller, J. Math. Phys. 10, 1004 (1969).

[59] M. Gutzwiller, J. Math. Phys. 11, 1791 (1970).

[60] M. Gutzwiller, Physica D 5, 183 (1982).

[61] W. Miller, J. Chem. Phys. 63, 996 (1975).

[62] H. Cartarius, J. Main, T. Losch, and G. Wunner, Phys. Rev. A 81, 063414 (2010).

[63] J. Main and G. Wunner, J. Phys. B: At. Mol. Opt. Phys. 27, 2835 (1994).

[64] W. Reinhardt, Annu. Rev. Phys. Chem. 33, 223 (1982).

[65] D. Delande, A. Bommier, and J. Gay, Phys. Rev. Lett. 66, 141 (1991).

[66] N. Moiseyev, Phys. Rep. 302, 212 (1998).

[67] T. Rescigno and V. McKoy, Phys. Rev. A 12, 522 (1975).

[68] N. Moiseyev, Non-Hermitian Quantum Mechanics (Cambridge University Press, Cambridge, 2011).

[69] P. Leboeuf and M. Saraceno, J. Phys. A: Math. Gen. 23, 1745 (1990).

[70] K. Husimi, Proc. Phys. Math. Soc. Jpn. 22, 264 (1940).

[71] E. Prugovečki, Ann. Phys. 110, 102 (1978).

[72] Y. Weissman and J. Jortner, J. Chem. Phys. 77, 1486 (1982).

[73] L. Ballentine, Quantum Mechanics: A Modern Development (World Scientific, Singapore, 1998).

[74] T. Bartsch, J. Main, and G. Wunner, J. Phys. A 32, 3013 (1999).

[75] J. Main and G. Wunner, Phys. Rev. A 55, 1743 (1997).

[76] M. Kuś, F. Haake, and D. Delande, Phys. Rev. Lett. 71, 2167 (1993). 